\title{
Oral dexamethasone decreases postoperative pain, swelling, and trismus more than diclofenac following third molar removal: a randomized controlled clinical trial
}

\author{
Carlos Alysson Aragão Lima ${ }^{1}$. Vinícius Tatsumoto Favarini ${ }^{1}$. \\ Alexandre Machado Torres ${ }^{2} \cdot$ Rogério Almeida da Silva $^{3} \cdot$ Fábio Ricardo Loureiro Sato ${ }^{4}$
}

Received: 28 August 2016 / Accepted: 29 May 2017 / Published online: 8 June 2017

(C) Springer-Verlag Berlin Heidelberg 2017

\begin{abstract}
Purpose The aim of this study was to compare the antiinflammatory potential of two pharmacotherapy protocols based on the parameters of pain, trismus, and swelling, after extraction of third molars.

Methods Thirty patients selected with symmetrical impaction of third molars were submitted to surgical procedures in both sides in different times. For one group, dexamethasone was used for 3 days, and for another group diclofenac sodium was also used for the same period. The main variables analyzed were the visual analogue pain scale (VAS), but others were also analyzed such as swelling and trismus, which were submitted to statistical analysis.

Results The results had no difference regarding the length of procedures $(p=0.986)$ and the pain in the immediate and 4-h postoperative period $(p=0.723$ and 0.541$)$. The rescue analgesic consumption was higher $(p<0.05)$ when using the protocol with diclofenac sodium. The variables mouth opening $(p<0.05)$ and swelling $(p<0.05)$ were significantly better
\end{abstract}

Fábio Ricardo Loureiro Sato

fabio.sato@ict.unesp.br

1 Resident of Oral and Maxillofacial Surgeon, Hospital Geral de Vila Penteado, São Paulo, Brazil

2 Oral and Maxillofacial Surgeon, Hospital Geral de Vila Penteado, São Paulo, Brazil

3 Oral and Maxillofacial Surgery Department, Hospital Geral de Vila Penteado, São Paulo, Brazil

4 Oral and Maxillofacial Surgery Area, State University of São Paulo-UNESP, College of Dentistry, São José dos Campos and Oral and Maxillofacial Surgeon, Hospital Geral de Vila Penteado, Av. Eng. Francisco José Longo, 777-12245-000, São José dos Campos, São Paulo, SP, Brazil when using the protocol with dexamethasone in the postoperative period.

Conclusions Medical protocol with the use of dexamethasone in the postoperative period was more effective in controlling pain, trismus, and swelling, after the extraction of third molars, when compared to diclofenac sodium.

Keywords Third molar surgery $\cdot$ Corticosteroids $\cdot$ Swelling . Pain · Trismus

\section{Introduction}

Inflammation is a vital process for the defense of the organism in tissue injury, such as surgical interventions, inflammatory mediators are released, resulting in vasodilation, increased vascular permeability, and other phenomena at tissue and cellular level which ultimately result in swelling, pain, temperature increase, erythema, and loss of function $[1,2]$.

The extraction of impacted third molars generates an inflammatory process due to trauma in soft and hard tissue during the surgery, resulting among others in pain, swelling, and trismus [3]. In view of increased comfort and improved quality of life for individuals undergoing extraction of impacted third molars, the modulation of the inflammatory process is necessary. Therefore, the use of non-steroidal anti-inflammatory drugs (NSAIDs) and/or corticosteroids is recommended.

Corticosteroids promote the removal of all phases of the inflammatory response, decrease capillary dilation, migration and total number of leukocytes, phagocytosis, inhibit the formation of granulation tissue and the production of vasoactive substances such as prostaglandins and leukotrienes, and retard the proliferation of fibroblasts and collagen synthesis. Corticosteroids also stimulate the production of lipocortin, which is a phospholipase A2 inhibitor protein [4]. The 
inhibition of phospholipase A2 enzyme interrupts the cascade of arachidonic acid and the synthesis of inflammatory mediators such as prostaglandins [5]. Various corticosteroids such as betamethasone, hydrocortisone, dexamethasone, and methylprednisolone are prescribed to control pain, swelling, and trismus [6].

The principle of preemptive analgesia is that therapeutic intervention must occur before the sensitization of pain receptors, helping to reduce the total concentration of inflammatory mediators in tissues [7]. The use of dexamethasone in order to reduce swelling, trismus, and postsurgical pain is supported by several studies $[6,8,9]$. It is believed that an intravenous dose of dexamethasone in the immediate preoperative phase has a great benefit in controlling swelling and trismus in the initial postoperative period. However, most corticosteroids, when used as a single dose in the preoperative period of oral surgeries, have no effects after $24 \mathrm{~h}$. In order to maintain their anti-inflammatory efficacy, the doses of corticosteroids must be kept for a period of 3 to 5 days, to maximize their benefits with minimum risk of removing the hypothalamic-pituitaryadrenal axis (HPA) [10].

The use of anti-inflammatory drugs varies between maxillofacial surgeons, based on many factors, such as personal experience and preference. This work was proposed to evaluate two medication protocols - a corticosteroid and a nonsteroidal anti-inflammatory drugs (NSAIDs) in the postoperative period of third molar surgery, considering the control of pain, swelling, and trismus after the surgery.

\section{Materials and methods}

\section{Study design}

This research was a clinical double-blind, randomized, and split-mouth study [11]. The research was submitted and approved by the Local Human Research Ethics Committees under CAA Protocol: 34824714.8.0000.5446. The study hypothesis is that corticosteroids, like dexamethasone, have a better control in pain, swelling, and trismus than the NSAID's when used in the postoperative period.

\section{Sample selection}

Fifty-two healthy patients were selected without deviations from normal vital signs, measured in the preoperative period, aged between 16 and 60 years old, who would be undergoing extraction of impacted third molars in the Oral and Maxillofacial Surgery Service.

Patients had to have four third molars impacted and with indication for extraction and with symmetry between the two sides, no pericoronitis or other inflammatory/infectious diseases at the time of surgery. All patients were informed by the main researcher about the research and those who agreed to participate signed the informed consent form. For the patients below 18 years old, the informed consent was signed by the adult responsible for those patients.

The study exclusion criteria were patients with a history of alcoholism, drug use, antihistamine drugs, antidepressants, cimetidine, or any drug that could interfere with the painful sensitivity of the patient. In addition, patients with systemic disorders such as diabetes, hypertension, heart disease, allergy to any component of the formula, sulfa drugs, and also pregnant women, nursing mothers, and children were excluded.

After exclusion of the patients that did not match the inclusion criteria or missed the follow-up control, the sample was composed of 30 patients. The CONSORT flow chart about sample selection is described in Fig. 1.

\section{Masking and randomization}

To ensure that the patient, the main researcher, the surgeon, and the statistician were unaware of what drug would be used postoperatively, dexamethasone (4 mg) and diclofenac sodium $(50 \mathrm{mg})$ were prepared in capsules of the same color and size and coded as drug no. 1 or drug no. 2 . The randomization procedure was performed by a researcher not involved in the evaluation and patient's surgeries, using sequentially numbered sealed envelopes. Each envelope had the combination of drugs to be used in the postoperative period, protocol 1 (with 1 drug) or protocol 2 (drug 2) and the side of surgery (right or left). For each enrolled patient, the researcher not involved in patient assessment and the surgery opened the envelope, informed the main researcher of the protocol to be

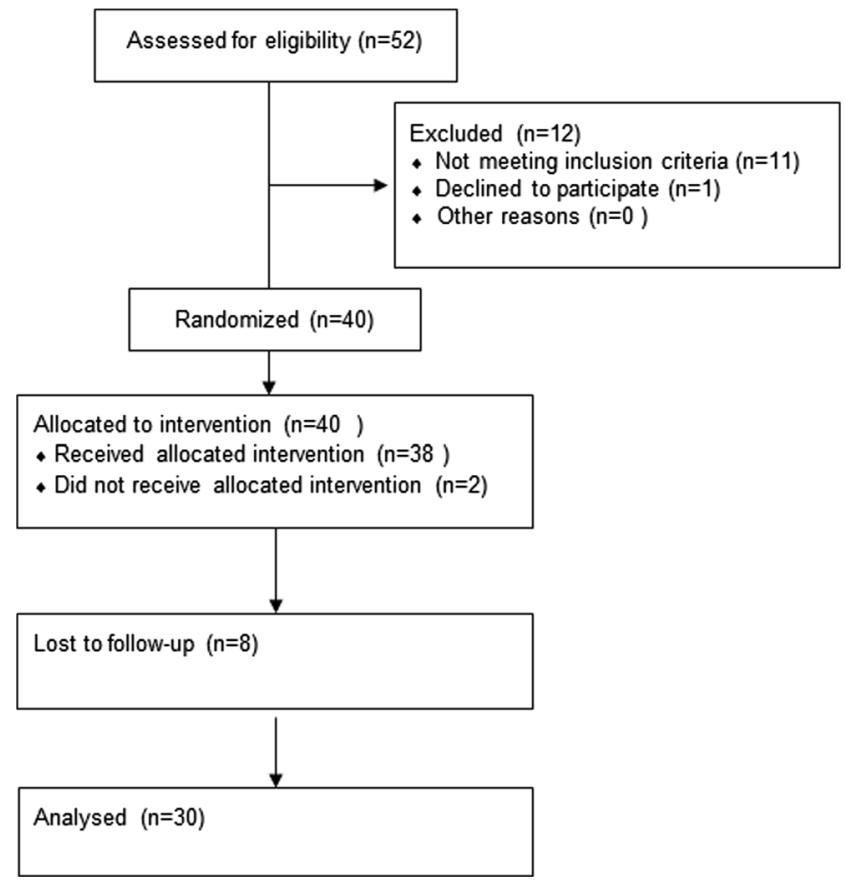

Fig. 1 CONSORT flow chart 


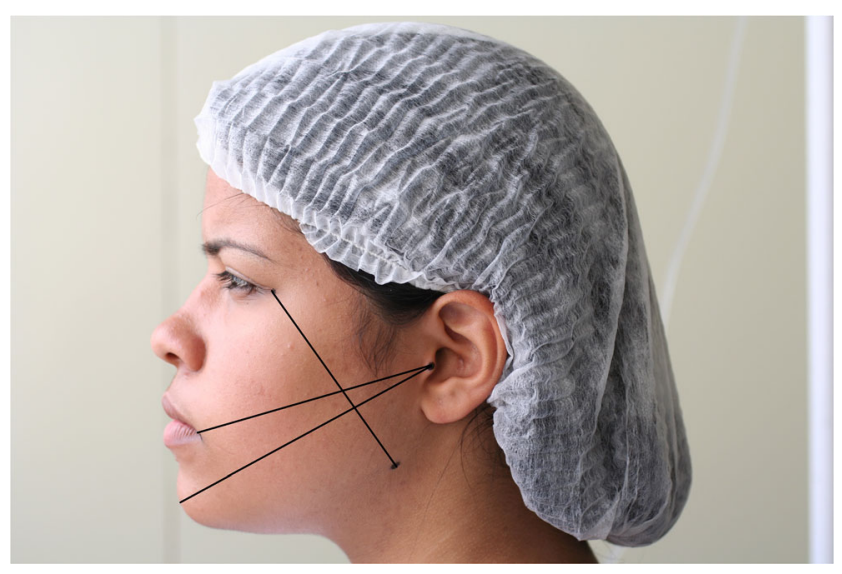

Fig. 2 Swelling measurements

used postoperatively, and the surgeon of the side to be operated. The second surgical procedure was performed on the contralateral side, using another medical protocol postoperatively, with an interval of 2 months between procedures.

The methodology of this study sought to follow the rules of the new CONSORT 2010 Statement [12] and is registered at ClinicalTrials.gov under number NCT02698306.

\section{Surgical procedures and drugs}

An hour before the surgery, all patients received $1 \mathrm{~g}$ of cefazolin intravenously as perioperative antibiotic prophylaxis, and as some procedures required a long time, the researchers decided to maintain the antibiotics postoperatively, using amoxicillin every $8 \mathrm{~h}$ after the procedure for 7 days for all procedures. No anti-inflammatory medication was given preoperatively to not impact on the postoperative medication evaluated in this research.
Table 1 Study sample characteristics

\begin{tabular}{ll}
\hline Study variable & Descriptive statistics \\
\hline Sample size & 30 patients \\
Gender & 19 females $/ 11$ males \\
Age & 21 years 6 months \pm 5 years and 8 months \\
\hline
\end{tabular}

With the patient in the supine position on the operating table, the surgeon performed the extra and intra oral antisepsis with chlorhexidine $0.2 \%$ and anesthesia of the inferior alveolar, lingual, buccal, posterior superior alveolar, and palatine nerves respecting the protocol described by Malamed [13], with lidocaine $2 \%$ and epinephrine 1:100,000. Initially, an incision was made at the alveolar crest along the mesial or distal aspect in the mandibular branch to reach the distolingual region of the second molar, followed by intrasulcular incision surrounding the second molar to the interdental area between the second and first molar. Afterwards, the surgeon raised the mucoperiosteal flap, performed an ostectomy procedure, sectioning of the tooth crown and/or roots when indicated for the case, tooth dislocation, dental avulsion, curettage, and copious irrigation of the cavity with saline solution $0.9 \%$ and absorbable polyglactin 910 sutures (Vycril 4.0-Ethicon Johnson \& Johnson do Brasil Ltda.-São Paulo). Then, the surgeon made an incision in the maxillary tuberosity along the mesial to distal aspect in order to reach the distal region of the second molar, followed by intrasulcular incision surrounding the second molar to the interdental area between the second and first molar, raised the total mucoperiosteal flap, ostectomy, tooth luxation, dental avulsion, curettage, and copious irrigation of the cavity with saline solution $0.9 \%$, absorbable polyglactin 910 wound sutures.
Fig. 3 Surgical time diclofenac $\times$ dexamethasone

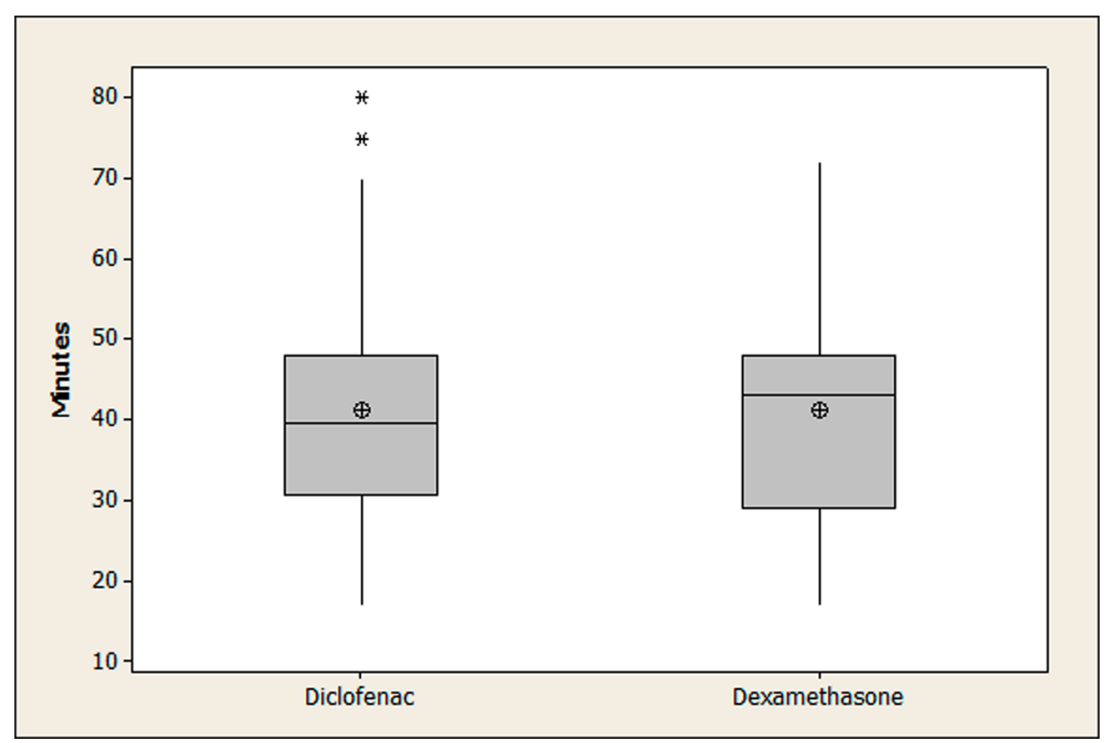


Table 2 Pain after surgery according to VAS scale

\begin{tabular}{|c|c|c|c|c|c|c|}
\hline & \multicolumn{2}{|c|}{ Immediate VAS } & \multicolumn{2}{|l|}{ VAS $4 \mathrm{~h}$} & \multicolumn{2}{|l|}{ VAS $8 \mathrm{~h}$} \\
\hline & Average & SD & Average & SD & Average & SD \\
\hline Diclofenac side & 0.33 & 0.8 & 4.17 & 2.36 & 3.97 & 2.92 \\
\hline Dexamethasone side & 0.27 & 0.64 & 3.77 & 2.28 & 2.53 & 2.03 \\
\hline$p$ value & 0.723 & & 0.541 & & $<0.05$ & \\
\hline
\end{tabular}

\section{Postoperative management}

After surgery, patients were divided into two groups based on medication protocol:

- Protocol 1: Use of dexamethasone every $8 \mathrm{~h}$ for 3 days and as rescue drug, in case of pain, dipyrone $(500 \mathrm{mg})$ that could be consumed every $4 \mathrm{~h}$.

- Protocol 2: Use of diclofenac sodium every $8 \mathrm{~h}$ for 3 days and as rescue drug, in case of pain, dipyrone $(500 \mathrm{mg})$ that could be consumed every $4 \mathrm{~h}$.

\section{Clinical assessments}

For both sides, the elapsed time of the surgical procedure was recorded, which was started at the time of the anesthetic administration and completed at the placement of the last suture.

The pain was measured from an analogue scale from 0 to 10 corresponding to the following: $0=$ no pain, 1 and $2=$ MILD, (easily tolerated), 3 to $5=$ MODERATE (tolerable discomfort), 6 to $8=$ strong (discomfort difficult to tolerate), and 9 to $10=$ VERY STRONG (unbearable), besides the pain score annotations at pre-set times (immediate postoperative period, 4 and $8 \mathrm{~h}$ postoperatively).

Fig. 4 Amount of dipyrone comparing the diclofenac $\times$ dexamethasone groups
Table 3 Difference in mouth opening

\begin{tabular}{llllll}
\hline & \multicolumn{2}{l}{$\mathrm{T} 2(\mathrm{~mm})$} & & & \multicolumn{2}{l}{$\mathrm{T} 3(\mathrm{~mm})$} & \\
\cline { 2 - 3 } \cline { 5 - 6 } & Average & $\mathrm{SD}$ & & Average & $\mathrm{SD}$ \\
\hline Diclofenac side & 15.87 & 7.46 & & 7.07 & 6.37 \\
Dexamethasone side & 8.33 & 3.79 & & 2.13 & 2.85 \\
$p$ value & $<0.05$ & & & $<0.05$ & \\
\hline
\end{tabular}

The patient filled a control questionnaire where the drugs consumed within $72 \mathrm{~h}$ was recorded.

The swelling was assessed by comparing the values of three facial lines: (1) the distance from the lateral corner of the eye to the gonial angle of the operated side; (2) the distance from the bottom edge of the tragus to the commissure of the mouth on the operated side; (3) the distance between the lower edge of the tragus to the soft pogonion of the operated side (Fig. 1). In three different times, those swelling measures were taken, evaluating the preoperative values (time 1) with postoperative values 2 nd day (time 2) and 7 th day (time 3 ) after surgery, measured by the third researcher (Fig. 2).

Trismus was assessed by comparing the values of the distance between the incisal edges of the upper and lower central incisors measured by a millimeter ruler. In three different times, the mouth opening were registered (preoperative values (time 1), postoperative values 2 nd day (time 2 ) and 7 th day (time 3) after surgery).

\section{Statistical analysis}

The data collected and tabulated in SPSS software v.16 (SPSS Inc., Chicago, 2007) were submitted to the Shapiro-Wilk

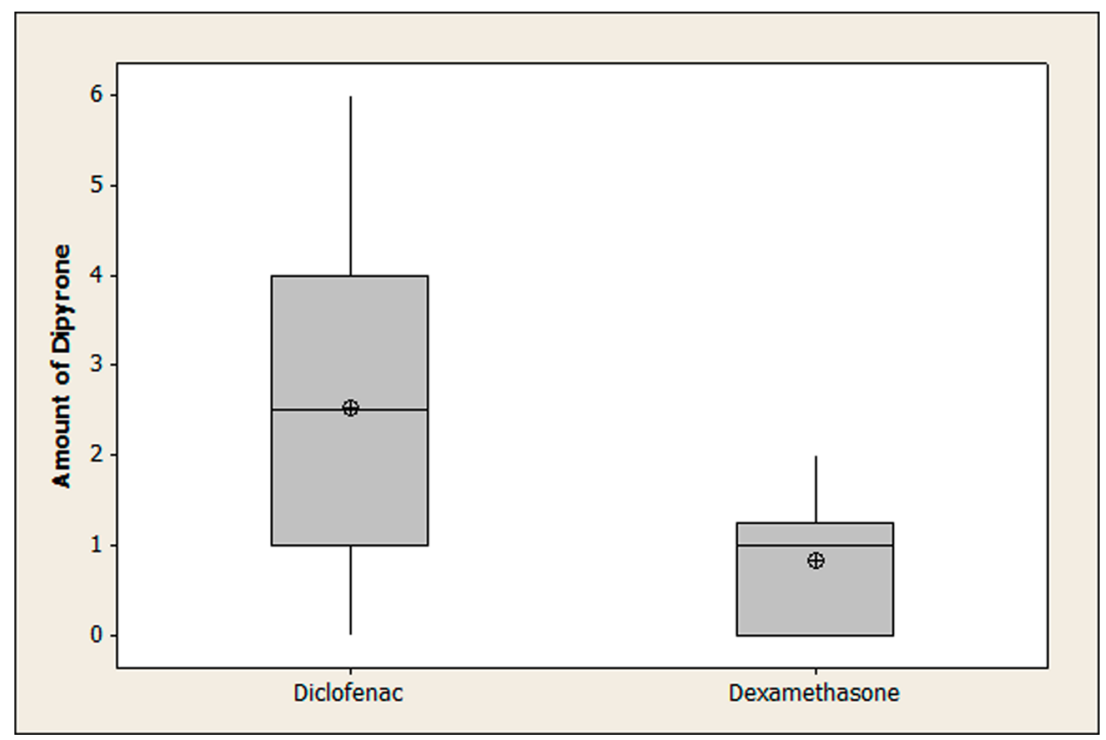


Table 4 Difference in postoperative swelling

\begin{tabular}{|c|c|c|c|c|c|c|c|c|c|c|c|c|}
\hline & \multicolumn{4}{|c|}{ Eye corner-gonion } & \multicolumn{4}{|c|}{ Tragus-lip commissure } & \multicolumn{4}{|c|}{ Tragus-pogonion } \\
\hline & \multicolumn{2}{|l|}{$\mathrm{T} 2(\mathrm{~mm})$} & \multicolumn{2}{|c|}{$\mathrm{T} 3(\mathrm{~mm})$} & \multicolumn{2}{|l|}{$\mathrm{T} 2(\mathrm{~mm})$} & \multicolumn{2}{|l|}{$\mathrm{T} 3(\mathrm{~mm})$} & \multicolumn{2}{|l|}{$\mathrm{T} 2(\mathrm{~mm})$} & \multicolumn{2}{|l|}{$\mathrm{T} 3(\mathrm{~mm})$} \\
\hline & Average & SD & Average & SD & Average & SD & Average & SD & Average & SD & Average & SD \\
\hline Diclofenac side & 5.3 & 2.39 & 2.53 & 1.5 & 6.77 & 3.04 & 3.43 & 1.85 & 6.97 & 3.08 & 3.6 & 2.09 \\
\hline Dexamethasone side & 2.03 & 1.29 & 0.33 & 0.48 & 2.93 & 1.59 & 0.77 & 0.85 & 2.9 & 1.42 & 0.733 & 0.74 \\
\hline$p$ value & $<0.05$ & & $<0.05$ & & $<0.05$ & & $<0.05$ & & $<0.05$ & & $<0.05$ & \\
\hline
\end{tabular}

normality test, and in view of the parametric data distribution, they were submitted to Student's $t$ test for each of the variables under study.

The sample power test was also made, and considering the difference of data between the averages and standard deviation for the pain variable (visual analogue scale, VAS) in the immediate postoperative period considering $10 \mathrm{~mm}$ as clinically relevant, with a sample of 30 patients for each group, the study has a sample power of $90.5 \%$.

\section{Results}

The demographics of the subject population for both groups are described in Table 1.

Surgical time was compared between the two sides of the patients. For such purpose, the statistical Student's $t$ test was used, and no difference was found between the sides $(p=0.986)$ (Fig. 3).

To evaluate the effectiveness of analgesia between the two medical protocols, it was compared to postoperative pain through the data obtained on a visual analogue scale in three stages, with a statistical difference found for the period of $8 \mathrm{~h}$ after the surgery, with minor VAS to the side on which dexamethasone was maintained postoperatively, and no difference in the immediate postoperative period and after $4 \mathrm{~h}$ (Table 2).

Still in the attempt to assess the analgesia of both protocols, the consumption of the rescue analgesic was evaluated in the postoperative period. According to the Student's $t$ test, a statistically significant difference was found $(p<0.05)$, with lower consumption to the side on which dexamethasone was maintained after the surgical procedure (Fig. 4). If considering the number of patients that did not have to use the rescue drug, for the dexamethasone groups we had 12 patients, and for the diclofenac 3 patients, also with a significant less usage of rescue drug in the dexamethasone group.

Regarding the variable mouth opening, the data were re-submitted to the Student's $t$ test, and there was a statistically significant difference $(p<0.05)$ in both measurements T2 (day 2) and T3 (day 7), with greater mouth opening to the side on which dexamethasone was maintained postoperatively (Table 3 ).

For the variable swelling, statistically significant differences were found in two times T2 and T3 $(p<0.05)$ in the three analyzed measures, with less swelling to the side on which dexamethasone was maintained after the surgery (Table 4).

\section{Discussion}

The perioperative administration of systemic corticosteroids is a pharmacological protocol commonly used to reduce postoperative morbidity in third molar surgery, but currently there is no well-accepted standard dosing regimen by the community of maxillofacial surgeons. Herrera-Briones et al. [14] concluded in their systematic review that more comparative studies with different corticosteroids are needed in order to establish the most effective regimen to control pain, swelling, and trismus after third molar extraction.

Corticosteroids have some analgesic effects resulting from anti-inflammatory and inhibitory action of inflammatory mediators such as prostaglandin $[15,16]$. In this study, regarding the amount of dipyrone used in the postoperative period of 7 days, on both sides, an increased consumption was noted on the side on which diclofenac was administered and a lower consumption on the side on which dexamethasone was maintained in the postoperative period. In addition, the VAS pain score was significantly lower when dexamethasone was maintained compared to diclofenac, indicating that dexamethasone was more effective in controlling pain.

However, there was no statistical difference when comparing the variable VAS pain in the immediate postoperative period, probably because the patients are still under the effects of local anesthetic used during surgery.

Regarding the mouth opening, the side on which diclofenac was used had a higher negative variation of inter-incisor distance when compared with the side on which dexamethasone was maintained postoperatively in both measured periods (time 2 and 3 ). Thus, trismus 
was higher when diclofenac sodium was used than when dexamethasone was maintained.

As for the swelling, in the three measures used (external angle-corner of his eye, tragus-pogonion, tragus-lip commissure) and in the two measured periods regarding the initial measures (time 1), there was a greater positive variation of the facial measurements when the diclofenac sodium was used compared to the side on which dexamethasone was maintained postoperatively. This denotes that swelling was significantly lower on the side on which dexamethasone was maintained.

Our results, as for swelling and trismus, corroborate the systematic review made by Markiewicz et al. [17], which suggest that perioperative administration of corticosteroids has a mild to moderate value in reducing swelling and trismus in the postoperative period of extraction of third molars. King et al. [10] reported that corticosteroids are effective agents in reducing the pain, swelling, and trismus after dentoalveolar surgery and the potential adverse effects with the use of corticosteroids depend on the intensity and duration of therapy, as the short period causes few adverse effects.

\section{Conclusion}

The medical protocol maintaining dexamethasone in the postoperative period was more effective in controlling pain, trismus, and swelling when compared to the protocol with diclofenac sodium.

\section{Compliance with ethical standards}

Conflict of interest The authors declare that they have no conflict of interest.

Funding No financial support for this study.

Ethical approval All procedures performed in studies involving human participants were in accordance with the ethical standards of the institutional and/or national research committee and with the 1964 Helsinki declaration and its later amendments or comparable ethical standards.

Informed consent Informed consent was obtained from all individual participants included in the study.

\section{References}

1. Laureano Filho JR, Maurette PE, Allais M, Milane C, Fernandes C (2008) Clinical comparative study of the effectiveness of two dosages of dexamethasone to control postoperative swelling, trismus and pain after the surgical extraction of mandibular impacted third molars. Med Oral Patol Oral Cir Bucal 13:129-132
2. Kocer G, Yuce E, Tuzuner OA, Dereci O, Koskan O (2014) Effect of the route of administration of methylprednisolone on oedema and trismus in impacted lower third molar surgery. Int J Oral Maxillofac Surg 43:639-632

3. Kulkarni D, Kshirsagar K (2011) Comparison of the efficacy of dexamethasone and methylprednisolone during post-operative period of surgical removal of impacted mandibular third molar-a clinical study. JIDA 5:683-686

4. Trummel CL (1998) Antiinflammatory drugs. In: Yagiela JA, Neidle EA, Dowd FJ, editors. Pharmacology and therapeutics for dentistry. Mosby; 297-319.

5. Timothy SC, William LF, Richard DD, John WM, Carroll AP, David FM (1998) A clinical evaluation of the analgesic efficacy of preoperative administration of ketorolac and dexamethasone following surgical removal of third molars. Anesth Prog 45:110-116

6. Gururaj A, Kirthi KR, Shivakumar HR, Bhushan J (2013) A randomized clinical trial to compare the efficacy of submucosal aprotinin injection and intravenous dexamethasone in reducing pain and swelling after third molar surgery: a prospective study. J Maxillofac Oral Surg 12:73-79

7. Rajesh G, Christopher RK, Laura EJ (2013) Does ketorolac have a preemptive analgesic effect? A randomized, double-blind, control study. J Oral Maxillofac Surg 71:2029-2034

8. Barroso BA, Lima V, Guzzo GC, Moraes RA, Vasconcellos MC, Bezerra MM, Viana FAL, Bezerra RCR, Santana GSM, FrotaBezerra FA, Moraes MO, Moraes MEA (2006) Efficacy and safety of combined piroxicam, dexamethasone, orphenadrine, and cyanocobalamin treatment in mandibular molar surgery. Braz J med Biol res 39:1241-1247

9. Simone JL, Jorge WA, Horliana ACRT, Carnaval TG, Tortamano IP (2013) Comparative analysis of preemptive analgesic effect of dexamethasone and diclofenac following third molar surgery. Braz Oral res 27:266-271

10. King K, Brar K, Kaltman JJS, Lopez E (2009) The use of corticosteroids and nonsteroidal antiinflammatory medication for the management of pain and inflammation after third molar surgery: a review of the literature. Oral Surg Oral med Oral Pathol Oral Radiol Endod 107:630-640

11. Dodson TB (2015) Writing a scientific paper is not rocket science! J Oral Maxillofac Surg 73:S160-S169

12. Moher D, Hopewell S, Schulz KF, Montori V, Gøtzsche PC, Devereaux PJ, Elbourne D, Egger M, Altman DG (2010) CONSORT 2010 explanation and elaboration: updated guidelines for reporting parallel group randomised trials. BMJ 340:869

13. Malamed S (2012) Handbook of local anesthesia, 6th edn. Mosby, St. Loius $6^{\text {a }}$ Edition

14. Herrera-Briones FJ, Sánchez EP, Botella CR, Capilla MV (2013) Update on the use of corticosteroids in third molar surgery: systematic review of the literature. Oral Surg Oral med Oral Pathol Oral Radiol 116:342-351

15. Dionne RA, Gordon SM, Rowan J, Kent A, Brahim JS (2003) Dexamethasone suppresses peripheral prostanoid levels without analgesia in a clinical model of acute inflammation. J Oral Maxillofac Surg 61:997-1003

16. Hargreaves KM, Costello A (1990) Glucocorticoids suppress levels of immunoreactive bradykinin in inflamed tissue as evaluated by microdialysis probes. Clin Pharmacol Ther 48:168-178

17. Markiewicz MR, Brady MF, Ding EL, Dodson TB (2008) Corticosteroids reduce postoperative morbidity after third molar surgery: a systematic review and meta-analysis. J Oral Maxillofac Surg 66:1881-1894 